\title{
Correction to: Hospital Readmission of Patients with Diabetes
}

\author{
Daniel J. Rubin ${ }^{1}$ \\ Published online: 13 March 2018 \\ (C) Springer Science+Business Media, LLC, part of Springer Nature 2018
}

\section{Correction to: Curr Diab Rep (2015) 15: 17 https://doi.org/10.1007/s11892-015-0584-7}

This article was originally published with errors that were introduced during the editing process. The corrected version of this article appears below.

\begin{abstract}
Hospital readmission is a high-priority health care quality measure and target for cost reduction. Despite broad interest in readmission, relatively little research has focused on patients with diabetes. The burden of diabetes among hospitalized patients, however, is substantial, growing, and costly, and readmissions contribute a significant portion of this burden. Reducing readmission rates of diabetic patients has the potential to greatly reduce health care costs while simultaneously improving care. Risk factors for readmission in this population include lower socioeconomic status, racial/ethnic minority, comorbidity burden, public insurance, emergent or urgent admission, and a history of recent prior hospitalization. Hospitalized patients with diabetes may be at higher risk of readmission than those without diabetes. Potential ways to reduce readmission risk are inpatient education, specialty care, better discharge instructions, coordination of care, and postdischarge support. More studies are needed to test the effect of these interventions on the readmission rates of patients with diabetes.
\end{abstract}

Keywords Hospital readmission · Inpatient diabetes· Transitional care Transitions of care $\cdot$ Risk factors $\cdot$ Discharge support

The online version of the original article can be found at https://doi.org/ $10.1007 / \mathrm{s} 11892-015-0584-7$

Daniel J. Rubin

djrubin@temple.edu

1 Section of Endocrinology, Diabetes, and Metabolism, School of Medicine, Temple University, 3322 N. Broad ST., Ste 205, Philadelphia, PA 19140, USA

\section{Introduction}

Hospital readmission is a high-priority health care quality measure and target for cost reduction, particularly within 30 days of discharge (30-day readmission, aka early readmission) [1-3]. Despite the broad interest in readmission, relatively little research has focused specifically on readmission of patients with diabetes [4-6]. The burden of diabetes among hospitalized patients, however, is substantial, growing, and costly, and readmissions contribute a significant portion of this burden. Reducing readmission rates among patients with diabetes has the potential to greatly reduce health care costs while simultaneously improving care. Recent research has provided some insight into the risk factors for readmission and the barriers to reducing readmission risk, as well as ways to mitigate that risk.

\section{Why Should We Care About Readmission Among Diabetic Patients?}

Many believe readmission rates reflect the quality of health care delivery, although this is debated [7]. Starting October 1, 2012, the Centers for Medicare and Medicaid Services began to decrease payments to hospitals that have greater-thanexpected 30-day readmission rates under the Hospital Readmission Reduction Program established by the Patient Protection and Affordable Care Act, reflecting an effort to motivate improvements in care while reducing costs [8]. In the current era of major health care reform, there is growing interest in hospital readmissions. There has been an exponential rise in the past decade of PubMed-cited articles published in this area, from less than 100 annually in the 1980 s to 1379 in 2013 [9].

Despite interest in readmissions, few studies have focused on readmissions among patients with diabetes [4-6]. There were only 85 publications listed on PubMed containing the search terms "hospital, readmission, and diabetes" in 2013 [9], and many of these publications are not relevant to this topic. 
The lack of attention paid to readmission of diabetic patients may reflect the fact that less than $2 \%$ of hospital discharges annually list a primary diagnosis of diabetes [10]. This statistic, however, belies the reality that the burden of diabetes among hospitalized patients is substantial, growing, and costly. Patients with diabetes currently represent about $9 \%$ of the US population [11], but they account for approximately $25 \%$ of hospitalizations (over eight million per year) $[10,12,13]$. The number and proportion of hospitalized patients with diabetes has increased steadily over the past two decades $[10,12,14]$, mirroring the increasing incidence and prevalence of diabetes in the general population $[11,15]$. While the overall 30-day readmission rate of hospitalized patients is $8.5-13.5 \%[16,17]$, the 30 -day readmission rate of diabetic patients is $14.4-22.7 \%$ [18, $19,20 \bullet, 21,22]$. Estimates of readmission rates beyond 30 days after hospital discharge are even higher, with over $26 \%$ of diabetic patients being readmitted within 3 months and $30 \%$ within 1 year $[23,24]$ In 2012, costs associated with the hospitalization of diabetic patients in the USA were $\$ 124$ billion, of which an estimated $\$ 25$ billion was attributable to 30-day readmissions assuming a $20 \%$ readmission rate $[12,25]$. Therefore, reducing 30 -day readmissions of patients with diabetes has the potential to greatly reduce healthcare costs while simultaneously improving care. A modest $5 \%$ reduction in the 30 -day readmission rate would result in 82,754 fewer admissions per year based on Agency for Healthcare Research and Quality Nationwide Inpatient Sample data from 2012, translating into an estimated annual cost savings of $\$ 1.2$ billion $[10,25]$. These data and the widespread interest in health care reform have prompted recent calls for more research on how to prevent readmissions in this important group of patients $[4,5,26]$.

In order for readmissions to be prevented, they must first be understood. A good understanding of the causes and risk factors for readmission is crucial. Readmission risk reduction interventions are more likely to be effective if they are informed by a solid understanding of the factors contributing to readmission risk. However, these interventions (discussed below) require human and financial resources. The resource requirements of interventions combined with the substantial prevalence of diabetes among hospitalized patients make interventions that attempt to reduce readmissions among all patients with diabetes impractical. Resources should therefore be focused on the highest-risk patients. Such high-risk patients do indeed exist. The Urban Diabetes Study, which included 291,752 discharges of diabetic patients in Philadelphia, PA, found that $10.5 \%$ of the patients were discharged at least five times over 8 years. This group of high utilizers accounted for $64 \%$ of all the discharges during the study period [18].

\section{Risk Factors for Readmission Among Patients with Diabetes}

\section{0-Day Readmission}

Studies of readmission risk factors among patients with diabetes can be grouped into those that focused on readmission within 30 days and those that examined readmission over longer time periods. Risk factors for 30-day readmission based on studies of at least 2000 patients are presented in Table $1[18,19,20 \bullet, 22,27,28,29 \bullet, 30,31]$. The following factors are associated with an increased risk of 30-day readmission and have been reported by at least two studies: male gender, comorbidity burden, hospital length of stay, government insurance vs. private or no insurance, emergent or urgent vs. elective admission, recent prior hospitalization, and being discharged against medical advice. Factors that are associated with an increased risk of 30-day readmission but have only been reported by one study or in abstract form include lower education level, being unemployed, living in an urban area vs. a remote rural area, lower income, receiving inpatient nutrition support, increased serum creatinine, lower serum hematocrit, being discharged with home health care or to another institution, the number microvascular or macrovascular diabetes complications, failing to recognize and document a diagnosis of diabetes, and, counterintuitively, having outpatient followup within 30 days of discharge. It is possible that sicker patients were more motivated to obtain follow-up than healthier patients and thus were more likely to be readmitted. There are conflicting data on the associations of age, race, ethnicity, proximity to the hospital, and A1c with 30-day readmission.

The few studies exploring an association of A1c with 30day readmission deserve a comment. A recently published single-center retrospective cohort study of 2265 hospitalized diabetic patients with an A1c $>9 \%$ did not find an independent association of baseline admission A1c with 30-day readmission [29•]. In contrast, a single-center retrospective cohort study of 1949 hospitalized patients with type 2 diabetes in a Boston-area primary care network found that the subgroup of patients with an admission A $1 \mathrm{c} \geq 8 \%$ had an approximately 20 $\%$ lower odds of 30-day readmission for each $1 \%$ increase in A1c [32•]. In further contrast, a retrospective cohort study of 880 diabetic and non-diabetic patients who underwent coronary artery bypass surgery found that a preoperative A1c $>6 \%$ was associated with a roughly twofold increase in the odds of readmission within 30 days after surgery [33]. Lastly, a retrospective cohort study of nearly 70,000 in patients with diabetes found that merely measuring the $\mathrm{A} 1 \mathrm{c}$ was associated with a decreased risk of 30-day readmission among patients with a primary diagnosis of diabetes but not those with other primary diagnoses [30]. This suggests that greater attention to diabetes reflected by A1c measurement may improve outcomes among patients hospitalized for diabetes. The association of A1c with 
Table 1 Risk factors for 30-day readmission of patients with diabetes

Risk factor Effect, if statistically significant [reference]

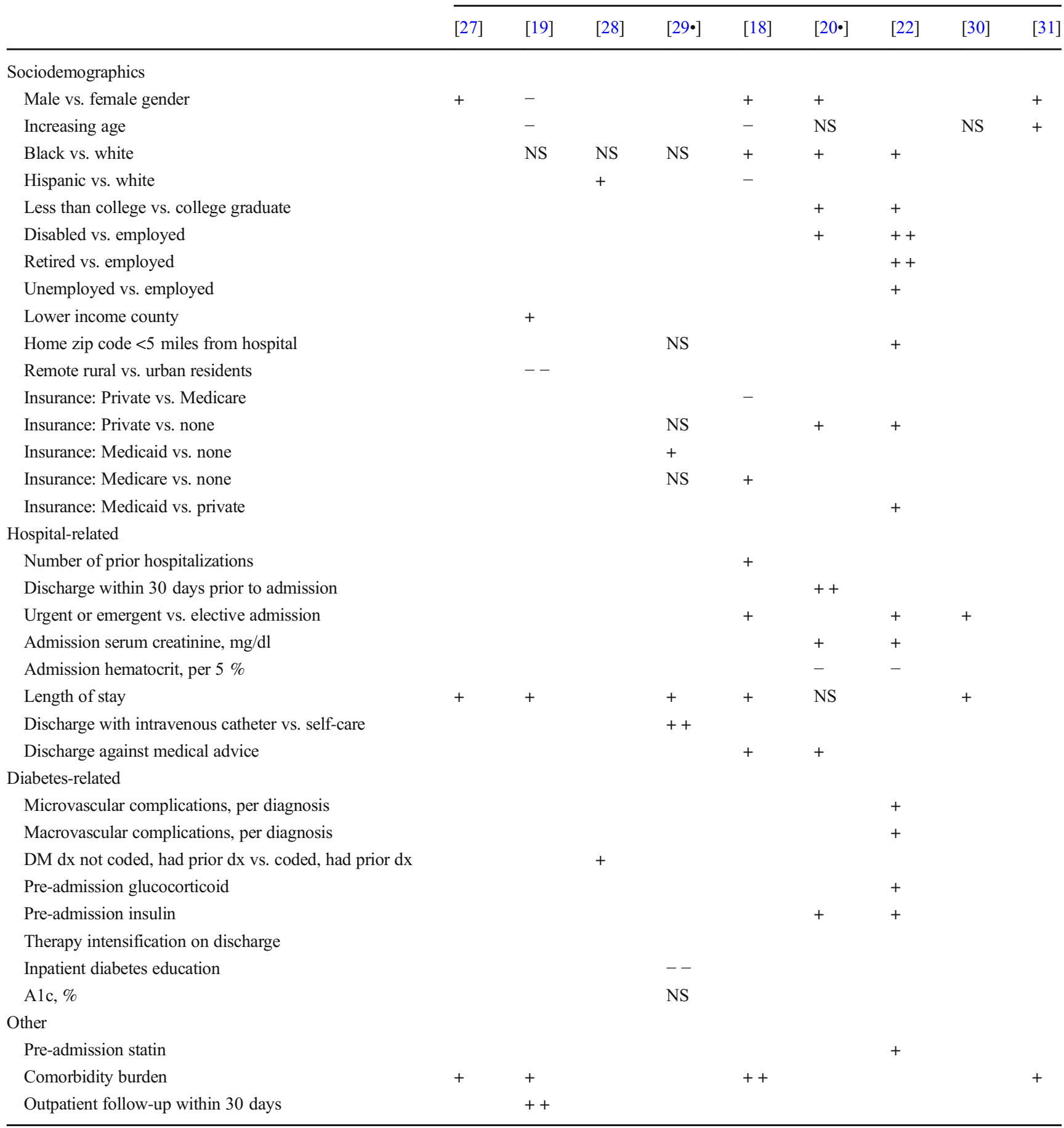

- odds ratio (OR) $0.75-0.99$ or not provided, $--\mathrm{OR}<0.75,+$ OR 1.01-2.00, + + OR $>2.00, D M$ diabetes mellitus, $d x$ diagnosis, NS not statistically significant

30-day readmission therefore remains unclear and may depend on the level of glycemic control and reason for hospitalization.

Most of the studies noted above used administrative databases to explore readmission risk factors or were not designed to identify reasons for readmission per se. In an effort to broaden the understanding of 30-day readmission among patients with diabetes, we performed a qualitative study [34•]. Based on thematic analysis of semi-structured interviews of adult in patients with diabetes who were readmitted within 30 days of 
hospital discharge, we identified five themes that contribute to readmission risk: (1) poor health literacy, i.e., lack of knowledge about diabetes and discharge instructions, (2) health system failure of the hospital discharge process and post-discharge support, (3) failure of factors that were expected to be protective, such as following the discharge instructions, being aware of medication changes upon discharge, and having help and social support at home, (4) social determinants of health impeding care, and (5) loss of control over illness. Most of the patients reported needing help with transportation, medications, and food. Patients generally denied actively exacerbating their condition prior to readmission and believed that being readmitted was out of their control. These findings suggest several points of intervention that will be discussed below.

\section{Predicting Early Readmission Risk}

While the work by other groups discussed thus far provides some insight into 30-day readmission risk factors for diabetic patients, none provides the ability to identify individual high risk patients at the point of care. We therefore developed the Diabetes Early Readmission Risk Index (DERRI), which is a multivariable logistic regression model that predicts the risk of all-cause 30-day readmission among hospitalized patients with diabetes [22]. The DERRI is based on a retrospective cohort of 17,595 adult patients with diabetes discharged between 2004 and 2012. Out of the 43 variables examined, there were 13 statistically significant predictors of 30-day readmission retained in the model (included in Table 1). The DERRI has good discriminatory ability (C-statistic 0.72 ) and is adequately calibrated (Hosmer-Lemeshow goodness-of-fit test $\mathrm{p}=0.41$ ). Early readmission risk in the highest tertile was 40 $\%$, accounting for $58 \%$ of all early readmissions. This early readmission risk index may be useful for targeting discharge support resources to those at higher risk.

\section{Readmission Beyond 30 Days After Discharge}

Not surprisingly, there is considerable overlap among risk factors for 30-day readmission of patients with diabetes and readmission more than 30 days after discharge, including higher comorbidity burden, public versus private insurance, lower income, length of stay, diabetes-related complications, and renal disease $[23,24,29 \cdot, 35,36]$. While data on the effect of race and ethnicity on 30-day readmission were mixed, multiple studies have shown that African American and Hispanic patients are at greater risk of readmission after 30 days than Caucasians $[23,29 \bullet, 35]$. In contrast to 30 -day readmission, patients living in urban areas are more likely to be readmitted after 30 days than rural patients $[23,37]$.

As with the data on 30-day readmission, the effect of glycemic control on later readmission is unclear. There is some evidence to support the hypothesis that poor glycemic control contributes to long-term readmission risk. First, a high A1c is associated with an increased risk for hospitalization for any cause and cardiovascular disease [38-40]. Second, insulin therapy post-discharge is associated with a lower risk of readmission at 1 year. In a study of patients with type 2 diabetes who used insulin before and during hospitalization, patients who continued insulin after discharge had lower risks of allcause and diabetes-related readmission within 12 months compared to patients who did not continue the insulin [41]. A companion study of insulin-naïve type 2 diabetes patients with baseline A1c levels $\geq 8 \%$ who initiated insulin during hospitalization found a trend for an association between insulin continuation and lower risk of readmission [42]. Among the subgroup of patients, however, with a baseline A1c $\geq 9 \%$, insulin continuation was significantly associated with a lower risk of all-cause (adjust hazard ratio [HR] 0.58 [0.36-0.93] 95\% CI) and diabetes-related readmission (adjusted HR 0.46 [0.23-0.87] 95\% CI).

In contrast to the evidence supporting a role of poor glycemic control in contributing to readmission risk, some data actually suggest that poor glycemia is associated with lower readmission risk. A one-arm pilot trial of individualized diabetes education and phone follow-up in 82 patients with an A1c $>9 \%$ found that lower baseline A1c was a weak predictor of readmission at 3 months but not at 6 months [43*0]. The same group performed a retrospective analysis of 2069 hospitalized diabetic patients with a baseline A1c $>9 \%$ and found that higher A1c was associated with lower risk of readmission within 6 months [29•]. Post hoc analysis revealed that higher A1c was only associated with lower readmission risk among those patients who received inpatient diabetes education, suggesting that patients with the worst glycemic control may stand to gain the most from education. Given the conflicting evidence regarding glycemic control and readmission risk, it is unclear if hyperglycemia is a marker for increased risk or a cause for readmissions. More research is needed to clarify this relationship.

\section{Diabetes as a Risk Factor for Readmission}

Patients with diabetes may be at higher risk of readmission than those without diabetes. In a study of 4769 medical patients, diabetes was associated with a statistically significant $40 \%$ increased risk of readmission within 90 days [44]. Diabetes is also associated with an increased risk of readmission in patients hospitalized for cardiac surgery [45, 46], heart failure [47, 48], acute myocardial infarction [49], stroke [50], or liver disease [51]. In contrast, recent studies have not found that diabetes is independently associated with an increased risk of 30-day readmission in medical patients or veterans $[52,53]$. Considered together, these data suggest that the effect of diabetes on readmission risk may vary by length of followup and by primary reason for hospitalization. 


\section{Barriers to Reducing Readmission Risk of Patients with Diabetes}

Having established that the risk of readmission of diabetic patients depends on multiple sociodemographic, medical, and process-related factors, and given the complexity of diabetes management, it is not surprising that multiple barriers to reducing readmission risk exist. Competing medical priorities may overshadow diabetes in the discharge planning process, especially among patients hospitalized for other reasons. For example, a survey of medical charts of diabetic patients at one academic teaching hospital found that $60 \%$ of discharge notes mentioned diabetes, and only $20 \%$ of discharges included a plan for diabetes follow-up [54]. Similarly, in our small qualitative study of readmitted diabetic patients, very few discharge instructions included diabetes-specific information other than medications [34•]. Despite the recommendation to intensify diabetes therapy in poorly controlled patients upon discharge [55], such intervention is often deferred to the outpatient setting [56]. Adding to the complexity of discharge planning, most patients have an inpatient regimen that is very different from their outpatient regimen, in accordance with current guidelines to discontinue non-insulin diabetes medications upon admission [55]. Furthermore, assessment of glycemic control and adjustment of diabetes therapy, especially insulin, should be performed much sooner after discharge than for most other chronic conditions [57••]. Lastly, providers may fail to provide outpatient follow-up within 30 days [56].

In addition to these health system factors, there are several patient-related factors that impede efforts to minimize readmission. Poor health literacy may be of particular importance given that diabetes requires more engagement by patients than many other chronic conditions [34•]. Patients with lower health literacy may not remember or understand their discharge instructions. In addition, misperceptions about diabetes control may persist despite inpatient diabetes education [58]. Poor health literacy, along with lack of financial resources and feeling powerless to control one's health, may contribute to medication nonadherence [34 $]$. Lack of financial resources, including transportation and health insurance, may impede the ability of patients to keep outpatient follow-up appointments [59]. Inadequate finances may also make it difficult to reach patients by phone, which limits the utility of phone-based interventions [43••, 58]. Although many of these factors apply to hospitalized patients in general, the multiple causes of readmission and barriers to reducing readmission risk of diabetic patients are complex and merit specific consideration.

\section{Strategies for Reducing Readmission Risk of Patients with Diabetes}

Although a number of trials have tested strategies to reduce readmission rates in various hospital populations, few have focused on patients with diabetes [60-66]. Rather, most of these studies have enrolled unselected hospitalized patients, those on medical services, the elderly, or those admitted for heart failure or other specific conditions. These trials have shown significant relative reductions in 30-day readmission risk ranging from 30 to $75 \%$. With one exception, all of the successful studies tested multi-component discharge bundles, suggesting that bundled interventions may realize an additive effect beyond that seen with a single intervention. Common components of these interventions were patient-centered discharge education, peri-discharge coordination of care, and post-discharge support. Whether these strategies could be applied successfully to patients with diabetes is unknown. Evidence supporting readmission risk reduction interventions specifically in diabetic patients comes from several retrospective and small prospective studies. The data from randomized controlled trials of inpatient diabetes specialty care are mixed. One trial in 179 hospitalized patients with diabetes found that daily rounds by a nurse diabetes educator and an endocrinologist improved inpatient glycemic control and reduced allcause readmission rates at 3 months from 32 to $15 \%$ $(p=0.01)$ [67]. In contrast, however, a randomized controlled trial in 300 diabetic inpatients found that a diabetes specialist nurse decreased length of stay but did not affect readmission rates at 1 year [68]. Readmissions before 1 year were not assessed in this trial, and it is possible that diabetes specialty care may only affect readmission risk less than 1 year after discharge.

Several studies have examined the effect of inpatient diabetes education on readmissions. A single-center retrospective cohort study in more than 2000 hospitalized diabetic patients with a baseline A1c $>9 \%$ found that inpatient diabetes education significantly reduced the risk of 30 -day readmission by $44 \%$ and 180-day readmission by $20 \%$ [29•]. In a one-arm pilot trial of inpatient diabetes education and post-discharge phone follow-up in 82 patients with a baseline A1c $>9 \%$, the 180-day readmission rate for uncontrolled hyperglycemia was $3.2 \%$, down from $28 \%$ of admissions due to hyperglycemia [43••]. Also in support of a beneficial effect of education, another randomized controlled trial of poorly controlled hospitalized patients with diabetes found that pharmacist discharge counseling improved medication adherence during the 150 days after discharge, increased the rate of follow-up visits, and lowered the A1c by $2 \%$. Although readmissions were not assessed in this study, it suggests some of the ways diabetes education may reduce readmission rates. Lastly, a randomized controlled trial in 65 diabetic patients admitted for hypoglycemia found that inpatient education, adjustment 
of medications, and discharge planning significantly reduced the incidence of 30- and 90-day readmissions for hypoglycemia while also reducing length of stay by more than 2 days [69].

In addition to inpatient diabetes specialty care and education, a few studies have explored the effect of intensifying diabetes therapy on readmission rates. A retrospective cohort study of 1949 hospitalized patients with type 2 diabetes found that intensifying diabetes therapy was not associated with 30-day readmission or emergency department visits [32•]. Among medical patients with a baseline A1c $\geq 8 \%$, however, intensification of therapy was associated with a statistically significant $77 \%$ lower odds of 30-day readmission/emergency department visits and a $1.8 \%$ lower A1c. Similarly, a retrospective cohort study of insulin naïve patients with type 2 diabetes initiated on insulin during hospitalization reported a trend toward lower 1-year readmission risk for those with baseline A1c levels $\geq 8 \%$ and a statistically significant lower risk in patients with baseline Alc levels $\geq 9 \%$ [42]. Likewise, a smaller retrospective cohort study in patients with type 2 diabetes and baseline A1c levels $\geq 10 \%$ found a similar beneficial effect of diabetes therapy intensification on 90-day readmission rates [70]. Thus, intensification of diabetes therapy upon discharge may reduce readmission risk among poorly controlled patients.

Another strategy for reducing readmission rates is dedicated outpatient support. A pilot randomized controlled trial in 100 diabetic medically indigent patients who lacked primary care reported that follow-up visits in a diabetes transitional care clinic 2 to 5 days after hospital discharge significantly decreased the rate of 90-day diabetes-related readmissions among the subgroup of patients originally admitted for diabetes [57••]. In the entire cohort, the transitional care clinic was associated with a nonsignificant reduction in 90-day readmissions ( 20 vs. $28 \%$ ). Another study focused on diabetes-related readmissions explored the effect of outpatient support provided to 115 patients with type 1 diabetes admitted for diabetic ketoacidosis [71]. In this nonrandomized trial, patients who consented to the intervention received at least four clinic visits annually in a dedicated diabetes treatment unit, monthly phone calls, and 24-h access to a diabetologist by phone. The intervention was associated with a significantly lower rate of readmissions for diabetic ketoacidosis over the 2year study period, a $3 \%$ lower A1c level, and a lower cost of diabetes-related care. Outpatient diabetes support therefore appears to decrease diabetes-related readmission risk.

Although not formally tested, there are a number of ways to improve the hospital discharge process and potentially reduce readmission risk. Our qualitative study of readmissions discussed above led to the conclusion that better communication of discharge instructions, involving patients more in medication reconciliation and scheduling follow-up appointments,
Table 2 Potential strategies to reduce readmission risk of diabetic patients

Inpatient diabetes education

For patients who are poorly controlled, new to diabetes or insulin, or have severe hypoglycemia

Inpatient diabetes consultation

For poorly controlled patients or those with a history of readmissions or recent severe hypoglycemia

Intensifying diabetes therapy based on admission A1c

Diabetes discharge instructions

Clearly written at an appropriate reading and health literacy level

Include information on follow-up appointments

Include contact information for providers

Using teach back to optimize patient communication

Follow-up planning

Involving patients in appointment scheduling

Assessing and addressing barriers to adherence

Post-discharge support by phone or outpatient visit within 1 week

Follow-up diabetes outpatient visit within 1 month after discharge

and assessing barriers to following the discharge plan may decrease the risk of readmission [34•]. Discharge instructions should be written clearly at an appropriate reading and health literacy level [4]. Discharge paperwork should also include ways for patients to contact post-hospital care providers and information on follow-up appointments, which are best scheduled prior to discharge. Communication with patients can be optimized with the teach-back method, which has been used successfully for outpatient diabetes care [72]. A summary of strategies to reduce readmission risk among patients with diabetes is presented in Table 2.

\section{Conclusions}

Hospital readmission of patients with diabetes is an important health care quality measure and driver of costs. Major risk factors for readmission include lower socioeconomic status, racial/ethnic minority, greater burden of comorbidities, public insurance, emergent or urgent admission, and a history of recent prior hospitalization. Certain hospitalized patients with diabetes may be at higher risk of readmission than those without diabetes. Multiple health system and patient-related barriers to reducing readmission rates exist. A mix of expert opinion and a handful of mostly small studies provide a number of potential strategies for reducing readmission risk, including inpatient education, specialty care, better discharge instructions, coordination of care, and postdischarge support. The diabetes-specific strategies such as diabetes education, intensifying therapy, and outpatient diabetes care tend to be more effective in poorly controlled patients and tend to reduce. 


\section{Compliance with Ethics Guidelines}

Conflict of Interest Daniel J. Rubin has received grant support from Merck for a subcontract for inpatient diabetes research.

Human and Animal Rights and Informed Consent This article does not contain any studies with human or animal subjects performed by any of the authors.

\section{References}

Papers of particular interest, published recently, have been highlighted as:

- Of importance

•- Of major importance

1. Axon RN, Williams MV. Hospital readmission as an accountability measure. JAMA. 2011;305(5):504-5.

2. Stone J, Hoffman G. Medicare hospital readmissions: issues, policy options and PPACA. Vol 7-5700, R40972: Congressional Research Service, Penny Hill Press; 2010.

3. Epstein AM. Revisiting readmissions - changing the incentives for shared accountability. N Engl J Med. 2009;360(14):1457-9.

4. Cook CB, Seifert KM, Hull BP, Hovan MJ, Charles JC, MillerCage V, et al. Inpatient to outpatient transfer of diabetes care: planing for an effective hospital discharge. Endocr Pract. 2009;15(3): 263-9.

5. Draznin B, Gilden J, Golden SH, Inzucchi SE, Baldwin D, Bode BW, et al. Pathways to quality inpatient management of hyperglycemia and diabetes: a call to action. Diabetes Care. 2013;36(7):1807-14.

6. Dungan KM. The effect of diabetes on hospital readmissions. J Diabetes Sci Technol. 2012;6(5):1045-52.

7. Benbassat J, Taragin M. Hospital readmissions as a measure of quality of health care: advantages and limitations. Arch Intern Med. 2000;160(8):1074-81.

8. Kocher RP, Adashi EY. Hospital readmissions and the affordable care act: paying for coordinated quality care. JAMA. 2011;306(16): 1794-5.

9. PubMed. US National Library of Medicine. National Institutes of Health. http://www.ncbi.nlm.nih.gov/pubmed. Accessed 29 Aug 2014.

10. HCUP Nationwide Inpatient Sample (NIS). Agency for healthcare research and quality (AHRQ). 2014. http://hcupnet.ahrq.gov/ HCUPnet.jsp (2012). Accessed 15 June 2014.

11. Centers for Disease Control and Prevention. National Diabetes Statistics Report: Estimates of Diabetes and Its Burden in the United States, 2014. Atlanta: U.S. Department of Health and Human Services; 2014.

12. HCUP Nationwide Inpatient Sample (NIS). Agency for healthcare research and quality (AHRQ); 2011. http://hcupnet.ahrq.gov/ HCUPnet.jsp. Accessed 11 Aug 2013.

13. Umpierrez GE, Isaacs SD, Bazargan N, You X, Thaler LM, Kitabchi AE. Hyperglycemia: an independent marker of inhospital mortality in patients with undiagnosed diabetes. J Clin Endocrinol Metab. 2002;87(3):978-82.

14. CDC. Number (in Thousands) of Hospital Discharges with Diabetes as Any-Listed Diagnosis, United States, 1988-2009. Centers for Disease Control and Prevention (CDC), National
Center for Health Statistics. http://www.cdc.gov/diabetes/ statistics/dmany/fig1.htm. Accessed 7 Jan 2013.

15. Increasing prevalence of diagnosed diabetes - United States and puerto rico, 1995-2010. MMWR Morb Mortal Wkly Rep. 2012;61(45):918-921.

16. Pennsylvania Health Care Cost Containment Council. Hospital Readmissions in Pennsylvania 2010. 2012:1-24. http://www. phc4.org/reports/readmissions/10/. Accessed 18 Dec 2012.

17. Friedman B, Jiang HJ, Elixhauser A. Costly hospital readmissions and complex chronic illness. Inquiry. 2008;45(4):408-21.

18. Robbins JM, Webb DA. Diagnosing diabetes and preventing rehospitalizations: the urban diabetes study. Med Care. 2006;44(3):292-6.

19. Bennett KJ, Probst JC, Vyavaharkar M, Glover SH. Lower rehospitalization rates among rural Medicare beneficiaries with diabetes. J Rural Health. 2012;28(3):227-34.

20. Rubin DJ, Handorf E, McDonnell M. Predicting early readmission risk among hospitalized patients with diabetes (7796). ENDO 2013: The Endocrine Society's 95th Annual Meeting. 2013. https://endo.confex.com/endo/2013endo/webprogram/Paper7796. $\mathrm{html}$. Accessed September 24, 2013. Identifies novel set of factors predictive of early readmission of patients with diabetes.

21. Chen JY, Ma Q, Chen H, Yermilov I. New bundled world: quality of care and readmission in diabetes patients. J Diabetes Sci Technol. 2012;6(3):563-71.

22. Rubin D, McDonnell M, Nelson D, Zhao H, Golden SH. Predicting hospital readmission risk with a novel tool: the diabetes early readmission risk index (DERRI). 1508-P. American Diabetes Association 74th Scientific Sessions, 06/2014. San Francisco, CA; 2014. Describes a novel tool to predict readmission risk of individual patients with diabetes prior to discharge.

23. Kim H, Ross JS, Melkus GD, Zhao Z, Boockvar K. Scheduled and unscheduled hospital readmissions among patients with diabetes. Am J Manag Care. 2010;16(10):760-7.

24. Jiang HJ, Stryer D, Friedman B, Andrews R. Multiple hospitalizations for patients with diabetes. Diabetes Care. 2003;26(5):1421-6.

25. ADA: Economic Costs of Diabetes in the U.S. in 2012. Diabetes Care. 2013.

26. Burke RE, Coleman EA. Interventions to decrease hospital readmissions: keys for cost-effectiveness. JAMA Intern Med. 2013;173(8):695-8.

27. Albrecht JS, Hirshon JM, Goldberg R, Langenberg P, Day HR, Morgan DJ, et al. Serious mental illness and acute hospital readmission in diabetic patients. Am J Med Qual. 2012;27(6):503-8.

28. Jiang HJ, Andrews R, Stryer D, Friedman B. Racial/ethnic disparities in potentially preventable readmissions: the case of diabetes. Am J Public Health. 2005;95(9):1561-7.

29. Healy SJ, Black D, Harris C, Lorenz A, Dungan KM. Inpatient diabetes education is associated with less frequent hospital readmission among patients with poor glycemic control. Diabetes Care. 2013;36(10):2960-7. Well-done retrospective study found that education is associated with lower risk of readmission

30. Strack B, DeShazo JP, Gennings C, Olmo JL, Ventura S, Cios KJ, et al. Impact of $\mathrm{HbA} 1 \mathrm{c}$ measurement on hospital readmission rates: analysis of 70,000 clinical database patient records. Biomed Res Int. 2014;781670:2014.

31. Zapatero A, Gomez-Huelgas R, Gonzalez N, Canora J, Asenjo A, Hinojosa $\mathrm{J}$, et al. Frequency of hypoglycemia and its impact on length of stay, mortality, and short-term readmission in patients with diabetes hospitalized in internal medicine wards. Endocr Pract. 2014:1-15.

32. Wei NJ, Wexler DJ, Nathan DM, Grant RW. Intensification of diabetes medication and risk for 30-day readmission. Diabet Med. 2013;30(2): e56-62. This retrospective study reports that intensifying diabetes therapy was associated with lower readmission risk 
33. Engoren M, Schwann TA, Habib RH. Elevated hemoglobin A1c is associated with readmission but not complications. Asian Cardiovasc Thorac Ann. 2014;22(7):800-6.

34. Rubin D, Donnell-Jackson K, Jhingan R, Golden SH, Paranjape A. Early readmission among patients with diabetes: a qualitative assessment of contributing factors. J Diabetes Complicat. 2014. This study presents a construct of 5 themes to explain readmission risk that complements findings from quantitative studies;28: 869-73. https://doi.org/10.1016/j.jdiacomp.2014.06.013.

35. Dungan KM, Osei K, Nagaraja HN, Schuster DP, Binkley P. Relationship between glycemic control and readmission rates in patients hospitalized with congestive heart failure during implementation of hospital-wide initiatives. Endocr Pract. 2010;16(6):945-51.

36. Smith DM, Giobbie-Hurder A, Weinberger M, Oddone EZ, Henderson WG, Asch DA, et al. Predicting non-elective hospital readmissions: a multi-site study. Department of veterans affairs cooperative study group on primary care and readmissions. J Clin Epidemiol. 2000;53(11):1113-8.

37. Cramer S, Chapa G, Kotsos T, Jenich H. Assessing multiple hospitalizations for health-plan-managed Medicaid diabetic members. J Healthc Qual. 2010;32(3):7-14.

38. Govan L, Wu O, Briggs A, Colhoun HM, Fischbacher CM, Leese GP, et al. Achieved levels of HbAlc and likelihood of hospital admission in people with type 1 diabetes in the Scottish population: a study from the Scottish diabetes research network epidemiology group. Diabetes Care. 2011;34(9):1992-7.

39. Menzin J, Korn JR, Cohen J, Lobo F, Zhang B, Friedman M, et al. Relationship between glycemic control and diabetes-related hospital costs in patients with type 1 or type 2 diabetes mellitus. J Manag Care Pharm. 2010;16(4):264-75.

40. Nichols GA, Rosales AG, Perrin NA, Fortmann SP: The association between different A1C-based measures of glycemia and risk of cardiovascular disease hospitalization. Diabetes Care. 2013.

41. Wu EQ, Zhou S, Yu A, Lu M, Sharma H, Gill J, et al. Outcomes associated with insulin therapy disruption after hospital discharge among patients with type 2 diabetes mellitus who had used insulin before and during hospitalization. Endocr Pract. 2012;18(5):651-9.

42. Wu EQ, Zhou S, Yu A, Lu M, Sharma H, Gill J, et al. Outcomes associated with post-discharge insulin continuity in US patients with type 2 diabetes mellitus initiating insulin in the hospital. Hosp Pract. 1995;40(4):40-48,2012.

43.• Dungan K, Lyons S, Manu K, Kulkarni M, Ebrahim K, Grantier C, Harris C, Black D, Schuster D. An individualized inpatient diabetes education and hospital transition program for poorly controlled hospitalized patients with diabetes. Endocr Pract. 2014;1-24. This one-arm pilot trial found that education and post-discharge support reduced diabetes-related readmission rates.

44. Phillips RS, Safran C, Cleary PD, Delbanco TL. Predicting emergency readmissions for patients discharged from the medical service of a teaching hospital. J Gen Intern Med. 1987;2(6):400-5.

45. Ferraris VA, Ferraris SP, Harmon RC, Evans BD. Risk factors for early hospital readmission after cardiac operations. J Thorac Cardiovasc Surg. 2001;122(2):278-86.

46. Stewart RD, Campos CT, Jennings B, Lollis SS, Levitsky S, Lahey SJ. Predictors of 30-day hospital readmission after coronary artery bypass. Ann Thorac Surg. 2000;70(1):169-74.

47. Keenan PS, Normand S-LT, Lin Z, Drye EE, Bhat KR, Ross JS, et al. An administrative claims measure suitable for profiling hospital performance on the basis of 30-day all-cause readmission rates among patients with heart failure. Circulation: Cardiovascular Quality and Outcomes. 2008;1(1):29-37.

48. Greenberg BH, Abraham WT, Albert NM, Chiswell K, Clare R, Stough WG, et al. Influence of diabetes on characteristics and outcomes in patients hospitalized with heart failure: a report from the organized program to initiate lifesaving treatment in hospitalized patients with heart failure (OPTIMIZE-HF). Am Heart J. 2007;154(2):277 e271-8.

49. Dunlay SM, Weston SA, Killian JM, Bell MR, Jaffe AS, Roger VL. Thirty-day rehospitalizations after acute myocardial infarctiona cohort study. Ann Intern Med. 2012;157(1):11-8.

50. Lichtman JH, Leifheit-Limson EC, Jones SB, Watanabe E, Bernheim SM, Phipps MS, et al. Predictors of hospital readmission after stroke: a systematic review. Stroke. 2010;41(11): 2525-33.

51. Berman K, Tandra S, Forssell K, Vuppalanchi R, Burton JR Jr, Nguyen $\mathrm{J}$, et al. Incidence and predictors of 30-day readmission among patients hospitalized for advanced liver disease. Clin Gastroenterol Hepatol. 2011;9(3):254-9.

52. Donzé J, Aujesky D, Williams D, Schnipper JL. Potentially avoidable 30-day hospital readmissions in medical patients: derivation and validation of a prediction model. JAMA Intern Med. 2013;173(8):632-8.

53. Shulan M, Gao K, Moore CD. Predicting 30-day all-cause hospital readmissions. Health Care Manag Sci. 2013;16(2):167-75.

54. Knecht LA, Gauthier SM, Castro JC, Schmidt RE, Whitaker MD, Zimmerman RS, et al. Diabetes care in the hospital: is there clinical inertia? J Hosp Med. 2006;1(3):151-60.

55. Umpierrez GE, Hellman R, Korytkowski MT, Kosiborod M, Maynard GA, Montori VM, et al. Management of hyperglycemia in hospitalized patients in non-critical care setting: an endocrine society clinical practice guideline. J Clin Endocrinol Metab. 2012;97(1):16-38.

56. Griffith ML, Boord JB, Eden SK, Matheny ME. Clinical inertia of discharge planning among patients with poorly controlled diabetes mellitus. J Clin Endocrinol Metab. 2012;97(6):2019-26.

57.• Seggelke SA, Hawkins RM, Gibbs J, Rasouli N, Wang C, Draznin B. Transitional care clinic for uninsured and medicaid-covered patients with diabetes mellitus discharged from the hospital: a pilot quality improvement study. Hosp Pract (1995). 2014;42(1):46-51. This RCT found that diabetes clinic visits within 5 days of discharge reduced diabetes-related readmission rates

58. Kimmel B, Sullivan MM, Rushakoff RJ. Survey on transition from inpatient to outpatient for patients on insulin: what really goes on at home? Endocrine practice : official journal of the American College of Endocrinology and the American Association of Clinical Endocrinologists. 2010;16(5):785-91.

59. Wheeler K, Crawford R, McAdams D, Robinson R, Dunbar VG, Cook CB. Inpatient to outpatient transfer of diabetes care: perceptions of barriers to postdischarge followup in urban African American patients. Ethn Dis. 2007;17(2):238-43.

60. Hansen LO, Young RS, Hinami K, Leung A, Williams MV. Interventions to reduce 30-day rehospitalization: a systematic review. Ann Intern Med. 2011;(8):155, 520-528.

61. Peikes D, Peterson G, Brown RS, Graff S, Lynch JP. How changes in Washington university's medicare coordinated care demonstration pilot ultimately achieved savings. Health Aff. 2012;31(6): 1216-26.

62. Watkins L, Hall C, Kring D. Hospital to home: a transition program for frail older adults. Prof Case Manag. 2012;17(3):117-23; quiz 124-115.

63. Evans RL, Hendricks RD. Evaluating hospital discharge planning: a randomized clinical trial. Med Care. 1993;31(4):358-70.

64. Koehler BE, Richter KM, Youngblood L, Cohen BA, Prengler ID, Cheng D, et al. Reduction of 30-day postdischarge hospital readmission or emergency department (ED) visit rates in high-risk elderly medical patients through delivery of a targeted care bundle. J Hosp Med. 2009;4(4):211-8.

65. Coleman EA, Parry C, Chalmers S, Min S. The care transitions intervention: results of a randomized controlled trial. Arch Intern Med. 2006;166(17):1822-8. 
66. Jack BW, Chetty VK, Anthony D, Greenwald JL, Sanchez GM, Johnson AE, et al. A reengineered hospital discharge program to decrease rehospitalization: a randomized trial. Ann Intern Med. 2009;150(3):178-87.

67. Koproski J, Pretto Z, Poretsky L. Effects of an intervention by a diabetes team in hospitalized patients with diabetes. Diabetes Care. 1997;20(10):1553-5.

68. Davies M, Dixon S, Currie CJ, Davis RE, Peters JR. Evaluation of a hospital diabetes specialist nursing service: a randomized controlled trial. Diabet Med. 2001;18(4):301-7.

69. Kampan P. Effects of counseling and implementation of clinical pathway on diabetic patients hospitalized with hypoglycemia. J Med Assoc Thail. 2006;89(5):619-25.
70. Lee PH, Franks AS, Barlow PB, Farland MZ. Hospital readmission and emergency department use based on prescribing patterns in patients with severely uncontrolled type 2 diabetes mellitus. Diabetes Technol Ther. 2014;16(3):150-5.

71. Maldonado MR, D'Amico S, Rodriguez L, Iyer D, Balasubramanyam A. Improved outcomes in indigent patients with ketosis-prone diabetes: effect of a dedicated diabetes treatment unit. Endocr Pract. 2003;9(1):26-32.

72. Schillinger D, Piette J, Grumbach K, Wang F, Wilson C, Daher C, et al. Closing the loop: physician communication with diabetic patients who have low health literacy. Arch Intern Med. 2003;163(1): 83-90. 